\title{
Accretion and Jet Power
}

\author{
Beverley J. Wills \\ Department of Astronomy, University of Texas at Austin, Texas, 78712
}

\begin{abstract}
In the first of a series of three lectures we discuss ways of measuring the power available to feed the jets in powerful FR II radio sources. For unobscured radio-loud QSOs we present evidence that this power is directly related to the UV-optical luminosity, or probably more accurately, to the power radiated through processes of accretion in a strong gravitational potential. It has been suggested on theoretical grounds that powerful radio jets are a necessary component of the central engine. It then follows, from the similarity of the optical-UV power output, spectral energy distribution, and emission-line spectra of radio-loud and radio-quiet QSOs, that radio-quiet QSOs have the same power available to feed jets as do radioloud QSOs. This then leaves us with the puzzle of why we do not see the powerful jets in radio-quiet QSOs.
\end{abstract}

\section{Introduction}

The enormous luminosity of QSOs $^{1}$ is believed to arise from accretion of gas through a large gravitational potential, over distance scales of $\sim 0.001$ pc, giving rise to the characteristic optical-ultraviolet-X-ray spectral energy distributions with $\mathrm{T} \sim 10^{4}-10^{5} \mathrm{~K}$ (the Big Blue Bump). Material is carried inwards in an accretion disk. Conservation of angular momentum requires the transport of angular momentum and energy outwards. One popular idea for achieving this (Königl \& Kartje 1995, but see Kundt \& Gopal-Krishna 1980) is that the disk, consisting of ionized plasma, is threaded by magnetic field lines that are twisted by the disk's rotation. Plasma flows out along the fields lines that then leave the disk perpendicular to the surface, allowing the plasma to be accelerated into collimated, relativistic outflows along the disk axis. Thus jets regulate the transport of angular momentum and energy; in this hypothesis they are a necessary component of the central engine, enabling the accreting fuel to spiral inwards.

The optical-UV-X-ray spectral energy distributions are remarkably similar for radio-loud and and radio-quiet QSOs (RLQs and RQQs). So also are the strong, broad emission-line spectra that arise in the surrounding high velocity gas of the broad line region (BLR), and the narrow-line spectra that

\footnotetext{
1 'QSO' refers to all luminous $\mathrm{AGN}\left(\mathrm{L} \gtrsim 10^{11} \mathrm{~L}_{\odot}, \mathrm{H}_{0}=100 \mathrm{~km} \mathrm{~s}^{-1} \mathrm{Mpc}^{-1}\right.$, and QSOs are called 'radio-loud' if $\mathrm{F}_{5 \mathrm{GHz}} / \mathrm{F}_{4400 \AA} \gtrsim 10$, where $\mathrm{F}$ is the rest frame flux-density in mJy; such strong radio emission is assumed to indicate powerful radio jets.
} 
arise from lower-velocity gas of the narrow line region (NLR) at greater nuclear distances $(\sim 1 \mathrm{pc}-\mathrm{Kpc})$; both the BLR and NLR are ionized by the $0.01-1 \mathrm{keV}$ photons from this central continuum source. These similarities strongly suggest the same type of central engine in RLQs and RQQs.

Even if we do not know the exact processes by which jets are formed, there are two empirical approaches that suggest that the power that feeds the jets is directly related to the power radiated by the central engine in the accretion process. We discuss these in $\S 2$ and $\S 3$.

\section{Jet Power, and Luminosity Relationships}

Two relations together show that jet power and accretion power (assumed to be represented by power radiated by QSO's Big Blue Bump) are strongly coupled - one between emission-line and optical-continuum luminosity, and the other between luminosity of emission lines and extended radio emission.

Yee (1980) and Shuder (1981) have shown that emission-line luminosities (e.g., $\mathrm{H} \alpha$ ) are closely proportional to the luminosity of the non-stellar, featureless continuum at $4800 \AA$ rest wavelength, over a range of more than $10^{5}$ in luminosity, for a heterogeneous assortment of narrow and broad line Seyfert and radio galaxies, RQQs and RLQs. They show that this relationship is consistent with photoionization of the emission-line regions by an extrapolation of the observed non-stellar continuum.

Rawlings \& Saunders (1991) report a close proportionality between the total kinetic power of the Kpc to Mpc-scale jets and narrow-line luminosity, for a complete sample of 3CR FR II radio galaxies. Despite their careful, detailed calculations of kinetic jet power, the result is not very different if simply the radio luminosity is used instead, provided any beamed core emission is excluded. The completeness of the sample is important, to demonstrate that the proportionality is not simply induced by the bias towards selecting sources of increasing radio and optical luminosity at higher redshift. Such an unbiased sample is not available for RLQs; however, if we do include radio and narrow emission-line data for FR I radio galaxies, and RLQs with $\mathrm{z}<1$, this relation extends over a range of $10^{4}$ in luminosity.

The first relation, between emission-line and continuum luminosity, shows that the ionizing continuum is closely related to the observed optical continuum, and that both are measured at least roughly, by the emission-line luminosity. This is useful because, in orientation-dust Unified Schemes, FR II radio galaxies are RLQs with the central engine hidden or partially hidden from the observer by a dusty torus or inner galaxy disk whose axis is parallel to the jet direction. In this case, even though the RLQ continuum and broad line region may be obscured, much of the extended gas is still illuminated and so narrow-line luminosity can apparently be used as a measure of the ionizing continuum and hence the accretion power. The second relation, between emission-line and extended radio luminosity, therefore shows that the 
kinetic power of the jet is directly related to the EUV-optical luminosity the accretion power. Moreover, Rawlings \& Saunders argue that the central engine channels a significant fraction of power into the jets compared with that radiated by accretion; this high efficiency implies a massive, spinning object that both powers the jets and controls the accretion rate.

Impressive as these relations are, the scatter in them is almost an order of magnitude. This scatter could be accounted for entirely by the uncertainties in determining jet power and by how well line luminosity measures the ionizing continuum. Significant scatter is certainly expected from variations in gas covering, optical depth to ionizing photons, dust reddening and obscuration. In other words, the true relationship between jet power and accretion power may actually be much tighter.

\section{Radio Core-dominance Relationships}

It is conventional to use the core dominance, $\mathrm{R}$, as a measure of the Dopplerboosting of radio synchrotron emission arising from relativistic flows at the base of the jet. $\mathrm{R}$ is defined as the ratio of flux-density of the compact radio source coincident with the QSO nucleus, to the (assumed isotropic) fluxdensity in the extended radio lobes, measured in the QSO rest frame. The nuclear flux density is typically measured with VLA resolutions of $\sim 1^{\prime \prime}$, and the nuclear source is usually unresolved at these resolutions. If the luminosity in the extended lobes is a good measure of the power available to feed the nuclear jets, and the bulk velocities at the base of the jet are similar from one RLQ to another, then R should be an indicator of the angle $\phi$, of the jet to the line-of-sight. On the simplest beaming hypotheses, $\mathrm{R}$ may range over factors of $\sim 10^{4}-10^{5}$, for blazars with jets within a few degress of the line of sight $($ small $\phi)$ to radio-galaxies with jets near the sky-plane $\left(\phi \sim 90^{\circ}\right)$.

Jets depend on synchrotron losses for their visibility, and there is strong evidence that their radio emission beyond several tens of Kpc from the nucleus depends increasingly on interactions with the host galaxy or intergalactic environment (Bridle et al. 1994). Their emission may be beamed even on scales of many Kpc. Also, they represent jet power averaged over millions of years. For these reasons extended radio luminosity may not be a good measure of present jet power, and so we looked for another way to normalize the beamed core flux-density (see Wills \& Brotherton 1995 for further discussion and references). We found that the use of optical luminosity for UV-excess FR II RLQs thought to have low reddening and negligible synchrotron contribution, significantly improved two relationships - those of $\mathrm{R}$ with jet angle and $\mathrm{R}$ with width (FWHM) of the broad $\mathrm{H} \beta$ emission line. The jet angle, $\phi$ is determined completely independently from measurements of superluminal motion and limits on inverse Compton scattered X-ray flux. These relationships are compared in Figs. 1 and 2. 
Fig. 1
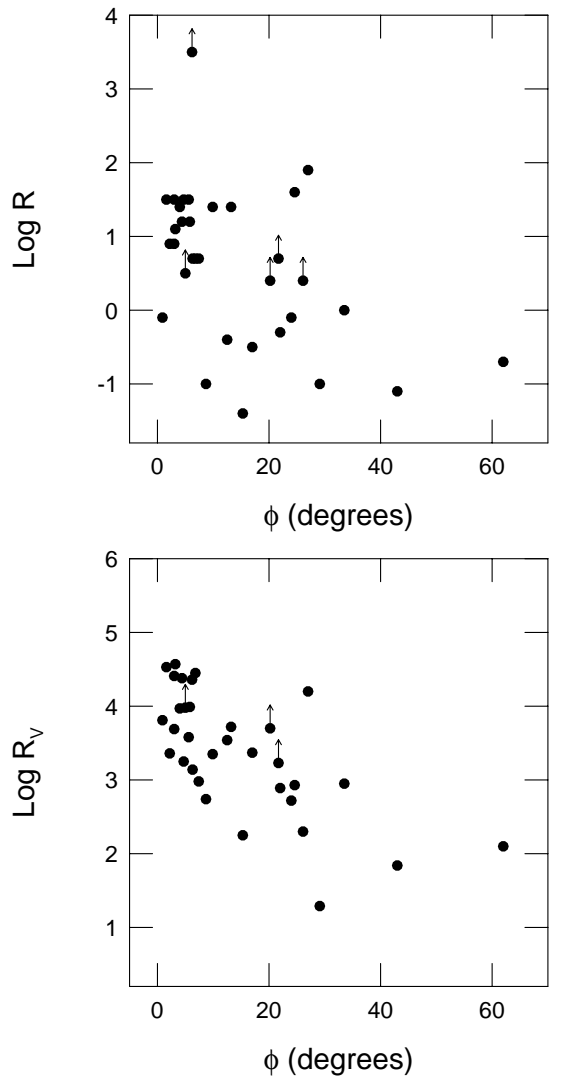

Fig. 2
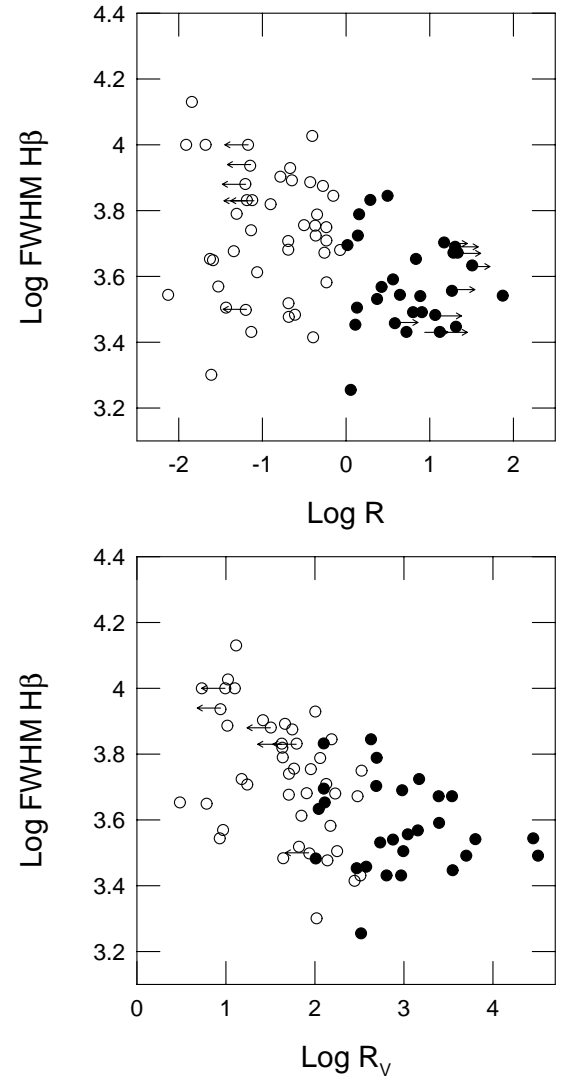

Fig. 1. Radio core-dominance vs. $\phi$, the angle of the beam to the line of sight. The 2 -tailed probability that these variables are unrelated decreased from $10^{-2}$ to $10^{-5}$ by using a rest-wavelength $\mathrm{V}$-band luminosity to normalize core-dominance $\mathrm{R}$.

Fig. 2. Radio core-dominance vs. the width of the broad $\mathrm{H} \beta$ line. Here, the 2-tailed probability that these variables are unrelated decreased from $4 \times 10^{-3}$ to less than $10^{-5}$ by using $\mathrm{R}_{V}$ instead of $\mathrm{R}$.

(Figures are adapted from Wills \& Brotherton 1995.) 


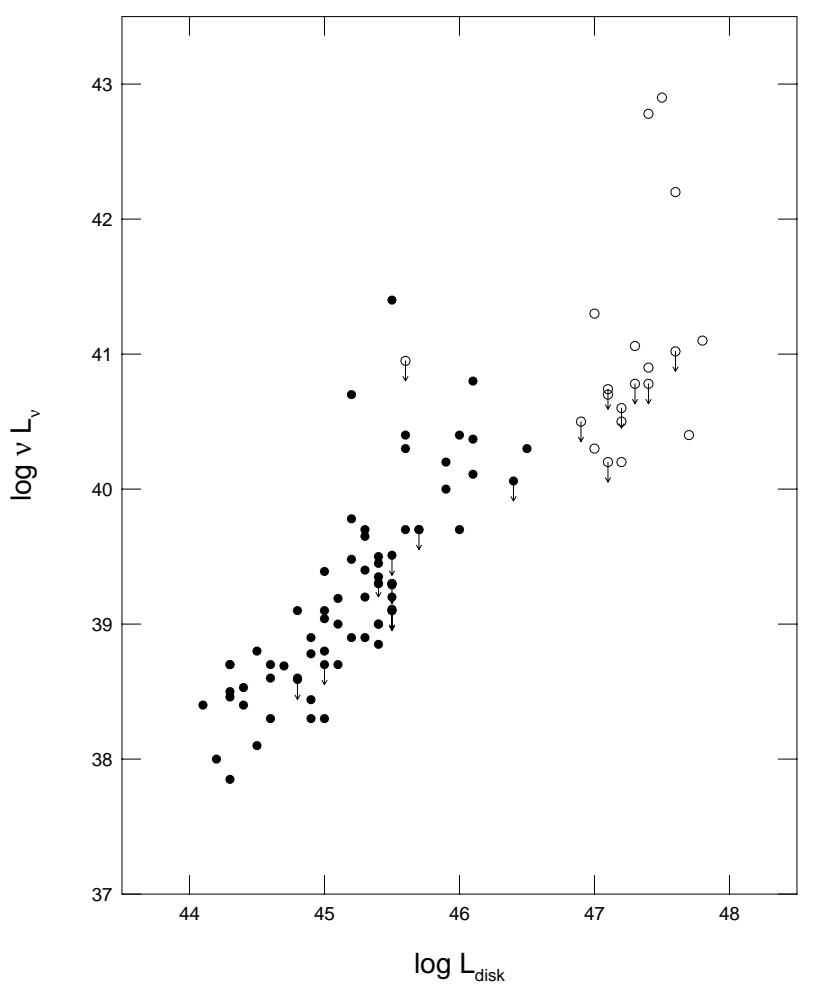

Fig. 3. Total radio luminosity vs. disk (accretion) luminosity, for radio-quiet QSOs. Data are for PG QSOs (the complete $\mathrm{z}<0.5$ sample is shown as filled circles, higher redshift QSOs are shown as open circles). The $\mathrm{L}_{\text {disk }}$ values are from Falcke et al. (1995), with radio luminosities updated using Kellermann et al. (1994). Many previous upper limits on $\nu L_{\nu}$ have become real measurements, showing even more convincingly the strong radio-UV luminosity relation.

\section{Radio Quiet QSOs}

Miller et al. (1992) and Falcke et al. (1995) show that there is a strong emission line vs. optical-UV continuum luminosity relationship for a complete, optically-selected PG sample, as also found for RLQs, showing a strong link between the observed and ionizing (EUV) continua. More importantly, despite the 100 times weaker radio luminosity, this complete sample of predominantly RQQs shows a closely proportional relationship between total $5 \mathrm{GHz}$ luminosity and accretion luminosity (Miller et al. 1993; Falcke et al. 1995). Fig. 3 shows this strong relationship from the paper by Falcke et al., updated with more recent radio luminosities from Kellermann et al. (1994), just for 
the PG QSOs. The relationship also suggests a narrow range of jet power to accretion power - providing further support for a real dichotomy between RLQs and RQQs.

We suggest that investigations of this dichotomy via the distribution of radio loudness for QSOs should be made in the radio luminosity vs. opticalUV luminosity plane rather than, as has sometimes been done, in the single radio-luminosity dimension, or even the single dimension of the ratio of radio luminosity to optical-UV luminosity. [Perhaps it would be better to calculate radio jet power instead of radio luminosity (Rawlings \& Saunders 1991), and a bolometric luminosity to represent accretion power, instead of optical-UV luminosity.]

\section{Summary and Discussion}

Previously-discovered relationships among emission line, optical continuum and extended radio luminosities indicate a proportionality between jet power and accretion power in high luminosity (FR II) sources. The improved correlations between core-dominance and jet angle, and between core-dominance and line width, when the beamed jet luminosity is normalized by the optical continuum, suggest an even stronger link on sub-parsec scales.

The similarity of the optical-to-EUV luminosities and spectra indicates the same accretion mechanism for the central engine of RLQs and RQQs

- this is also suggested by the similar relationship between extended radio luminosity and optical luminosity, albeit at very different radio luminosities. These similarities, together with the conclusion that this relationship is a strong proportional one for QSOs with powerful radio jets, suggest that the same power is potentially available to feed radio jets in RQQs. It is therefore a real puzzle that $90 \%$ of QSOs are radio-quiet.

The conclusion that radio jet power depends directly on ultraviolet-optical luminosity may seem to contradict the established belief that there is a very wide spread in extended radio luminosity for a given optical luminosity. It now takes on new meaning to ask whether there is a real dichotomy in radio loudness between RLQs and RQQs. If the central engines are identical, the differences in (unbeamed) jet brightness must have an extrinsic cause and therefore be dependent on the environment beyond sub-parsec scales. The question then becomes one of the distribution of environment conditions, and whether there is a discontinuity in the environmental conditions between RLQs and RQQs. An emission line approach to this will discussed in the next chapter. 


\section{References}

Bridle A. H., Hough D. H., Lonsdale C. J., Burns J. O., Laing R. A. (1994): Deep VLA Imaging of Twelve Extended 3CR Quasars. AJ 108, 766-820

Emmering R. T., Blandford R. D., Shlosman I. (1992): Magnetic Acceleration of Broad Emission Line Clouds in Active Galactic Nuclei. ApJ 385, 460-477

Falcke H., Malkan M. A. and Biermann P. L. (1995): The jet-disk Symbiosis II. Interpreting the radio/UV Correlations in Quasars. A\&A 298, 375-394

Kellermann K. I., Sramek R. A., Schmidt M., Green R. F., Shaffer D. B. (1994): The Radio Structure of Radio-loud and Radio-quiet Quasars in the Palomar Bright Quasar Survey. AJ 108, 1163-1177

Königl A., Kartje J. F. (1994): Disk-Driven Hydromagnetic Winds as a Key Ingredient of Active Galactic Nuclei Unification Schemes. ApJ 434, 446-467

Kundt W., Gopal-Krishna (1980): Extremely Relativistic Electron-positron Twinjets form Extragalactic Radio Sources. Nature, 288, 149-150

Miller P., Rawlings S., Saunders R., Eales S. (1992): A Spectrophotometric Study of BQS Quasars. MNRAS 254 93-110

Miller P. Rawlings S., Saunders R. 1993: The Radio and Optical Properties of the z $<0.5$ BQS Quasars. MNRAS 263, 425-460

Rawlings S., Saunders R. (1991): Evidence for a Common Central Engine Mechanism in all Extragalactic Radio Sources. Nat 349, 138-140

Shuder J. M. (1981): Emission-line-Continuum Correlations in Active Galactic Nuclei. ApJ 244, 12

Wills B. J., Brotherton M. S. (1995): An Improved Measure of Quasar Orientation. ApJ 448, L81-L84

Yee H. K. C. (1980): Optical continuum and emission-line luminosity of active galactic nuclei and quasars. ApJ 241894 